\title{
Dynamic interaction between glioma cells and their microenvironment: targeting Rac1 as a therapeutic approach
}

\author{
Georgina A. Cardama, Pablo Lorenzano Menna \\ Laboratory of Molecular Oncology, National University of Quilmes, Bernal, CP 1876, Buenos Aires, Argentina
}

Correspondence: Pablo Lorenzano Menna

E-mail: plmenna@unq.edu.ar

Received: June 12, 2015

Published online: July 29, 2015

\begin{abstract}
Rac1 has been described as a major player in glioma invasion and progression. The development of novel anti Rac1 inhibitors, such as 1A-116, provides a targeted rational approach to counteract glioma progression. This therapeutic strategy could affect the glioma cell behavior as well as its interplay with the microenvironment. Further studies are needed to validate the effectiveness of this novel molecules and their use in glioma progression.
\end{abstract}

Keywords: Glioma; Rho GTPases; Rac1; invasion; rational design; targeted therapy

To cite this article: Georgina A. Cardama, et al. Dynamic interaction between glioma cells and their microenvironment: targeting Rac1 as a therapeutic approach. Can Cell Microenviron 2015; 2: e866. doi: 10.14800/ccm.866.

\section{Introduction}

Gliomas are brain neoplasms with a glial cell origin and represent the most common primary tumors of the central nervous system in humans, with a mean survival of less than 1 year. The current treatment for gliomas includes the use of surgery, radio- and chemotherapy ${ }^{[1]}$. Despite their limited metastatic potential outside the central nervous system, gliomas are characterized by their high invasion potential throughout the brain. Migration and invasion of glioma cells is one of the most challenging processes that mainly contribute to the high frequency of tumor recurrence and tumor progression [2]. Consequently, new therapeutic approaches are essential to improve treatment outcome.

A key group of molecules involved in glioma cell invasion are Rho-GTPases. These proteins are molecular switches that cycle between two conformational states: an inactive GDP-bound form and an active GTP-bound form. This cycle is highly regulated by guanine nucleotide exchange factors
(GEFs) that catalyze nucleotide exchange and mediate activation, and GTPase-activating proteins (GAPs), that stimulate GTP hydrolysis and inactivate the GTPase ${ }^{[3]}$. The active GTP-bound state binds preferentially to downstream effector proteins and actively transduces signals ${ }^{[4]}$.

Rac1 is one of the most studied members of the family and controls fundamental cellular processes such as actin cytoskeleton reorganization; affecting endocytosis and trafficking, cell cycle progression, cell adhesion and migration. Accumulating evidence indicates that Rac1 is overexpressed and/or hyperactivated in a wide range of nervous system tumors. In astrocytomas, Rac1 is overexpressed in high-grade tumors. Notably, the plasma membrane localization of Rac1 observed in a subset of glioblastoma multiforme tumors, but not in low-grade astrocytomas or non-neoplasic brain, indicates that Rac1 is hyperactivated in glioblastomas ${ }^{[5,6]}$. Importantly, Rac1 was identified as a key molecule involved in the invasive behavior of gliomas. Many GEFs have been found to 
contribute to Rac1 activation and mediate glioma cell motility, such as Ect2, Vav3, Trio, Dock180, among others ${ }^{[7}$, ${ }^{8]}$.

Due to their key role in many transforming events in different cancer types, Rho GTPases are therefore attractive and validated targets for anticancer therapies ${ }^{[9]}$. Recently, we identified ZINC69391 as a small molecule that inhibited Rac1-GEF interaction [10, 11]. We further developed novel analogs using ZINC69391 as the parental compound and we showed that $1 \mathrm{~A}-116$ analog is a more potent Rac1 inhibitor. Both, ZINC693291 and 1A-116 had no effect on Cdc42 GTPase activation, a close-related Rho GTPase member of Rac1.

Two structurally unrelated families of GEFs have been described so far: the classical $\mathrm{Dbl}$ and the atypical Dock180-related families. The mechanism involving Trp56 by which many Dbl-GEFs bind and activate Rac1 has been known for more than a decade ${ }^{[12,13]}$. More recently, it has been described that Dock180 also shares the same residue as a determinant for specific recognition for Rac1 [14]. In support of this, we already showed that ZINC69391 was able to interfere Rac1-Tiam1 (a dbl-family GEF) recognition and ZINC69391 also blocks Rac1-Dock180 interaction. Dock180 has been characterized to contribute to the enhancement of glioma cell migration and invasion via Rac1 activation. Moreover, Dock180 was found to be involved in the signaling pathways that mediate PDGFR $\alpha$ - and EGFRvIIIdriven tumorigenesis and invasion in glioma, via Rac1 activation ${ }^{[15,16]}$. In line with the evidence indicating that ZINC69391 blocks Rac1-Dock180 interaction, ZINC69391 effectively reduced endogenous Rac1 activation levels. We also examined the effect of ZINC69391 treatment on Pak1 phosphorylation. Pak1 is widely expressed in a variety of normal tissues, and its expression is significantly increased in different cancers. In malignant glioblastoma, immunohystochemical analysis showed that phosphorylated Pak1 levels in the cytoplasm of glioma specimens correlated with shorter survival time in patients ${ }^{[17]}$. Our results present that treatment of LN229 cells with ZINC69391 reduced Pak1 phosphorylation in a concentration-dependent manner upon EGF stimulation. These results demonstrate that ZINC69391 inhibits Dock180-Rac1-Pak1 signaling pathway on glioma cells in vitro.

In agreement with observations that used expression of dominant-negative Rac1 to inhibit proliferation and survival of glioma cell lines [18], ZINC69391 treatment showed antiproliferative activity in LN229 and U-87 MG cell lines. This inhibition was associated with a cell cycle arrest in G1 phase. We also show an increase in subG0 cell population, suggesting apoptosis induction. To extent our observations, we evaluated the proapoptotic activity of ZINC69391. Annexin V and TUNEL studies established that ZINC69391 induces apoptosis on glioma cell lines. These findings are in agreement with observations that used recombinant adenoviruses expressing dominant negative Rac1 on glioma cell lines and glioblastoma primary cultures to suppress Rac1 activity ${ }^{[19]}$. Senger et al, showed that Rac1 inhibition induces apoptosis in glioma cells but not in normal human astrocytes.

As previously mentioned, diffuse cell migration and invasion are key properties of malignant gliomas. This invasive behavior has major therapeutic implications, since it is the major driver of glioma recurrence and therapeutic failure ${ }^{[1]}$. Surgery is unable to cure patients even when the lesions arise in areas in which wide surgical resection is possible, since glioma cells infiltrate throughout the brain. Rac1 GEFs such as Ect2, Vav3 and Trio have been implicated in glioblastoma cell invasion ${ }^{[20]}$ and Dock180 in EGFRvIII-driven invasion. This shows that Rac1 axis is important for glioma motility. In agreement with observations that used expression of dominant-negative Rac1 and siRNA Rac1 to interfere with glioma cell invasion, our data indicate that ZINC69391 modulates actin cytoskeleton dynamics inhibiting cell migration and having a significant impact on cell invasion in vitro.

Our data also show the increased potency of 1A-116 analog compared to parental ZINC69391 compound. This analog was rationally designed using ZINC69391 as a lead structure and was selected from a group of analogs because better docking scores correlated with an improved in vitro and in vivo potency on an aggressive breast cancer model. Importantly, ZINC69391 and analog 1A-116 showed to be more potent inhibitors than the described Rac1 inhibitor NSC23766 on cancer cells in vitro ( $\mathrm{IC}_{50}$ values: NSC23766: 140 $\mu \mathrm{M}, \mathrm{ZINC69391:} 61 \mu \mathrm{M}, 1 \mathrm{~A}-116: 4 \mu \mathrm{M})^{[10]}$.

Interestingly, 1A-116 analog presented also an improved antiproliferative activity compared to the parental analog on glioma cells and this antiproliferative activity showed to be Rac1 dependent using siRNA probes. We also show that 1A-116 analog is a more potent anti-invasive compound using Matrigel coated-transwells, a key feature for an anticancer agent for glioma treatment.

Taken together, our data provides evidence that Dock180/Rac1/Pak1 signaling pathway is downregulated by ZINC69391 compound leading to inhibition of cell proliferation and cell invasion on glioblastoma cell lines. Treatment with ZINC69391 arrested glioma cells in G1 phase, triggered apoptosis and modulated actin cytoskeleton dynamics, resulting in inhibition of cancer cell motility. We 
also show that $1 \mathrm{~A}-116$ analog inhibits in vitro proliferation and invasion more efficiently.

We focused our data so far on the impact of Rac1 inhibition in the tumor cell behavior. But this is only part of the complex mechanism governing the biology of gliomas. Tumor progression is orchestrated not only by glioma cells, but also by the tumor microenvironment. This complex network of different cell types, soluble factors, signaling molecules and extracellular matrix components are a critical aspect of the disease mechanism. Tumor-associated cells, such as endothelial cells, pericytes, microglia, astrocytes and macrophages have vital roles in the course of glioma progression. This complex interplay also affects therapeutic efficacy, since many of these cell types protect cancer cells from anti-cancer agents ${ }^{[21]}$. This represents an additional challenge for glioma therapeutics and its effect on patient outcome.

As mentioned before, GBM tumors are intrinsically invasive and infiltrative. Cancer cells utilize different strategies for migration, namely glioma dispersal into the surrounding tissue involves detachment from the primary location, attachment to and degradation of the extracellular matrix (ECM), and finally migration. Rac1 has historically been implicated in the molecular mechanisms of cell migration, affecting mainly actin cytoskeletal reorganization. But Rac1 regulation is also associated to matrix metalloproteinase 2 (MMP-2) secretion and activity ${ }^{[22]}$. Further, high levels of MMP-2 in tissues are associated with tumor cell growth and invasion ${ }^{[23]}$.

Apart from the infiltrative and invasive phenotype, malignant brain tumors are among the most vascularized tumors in humans ${ }^{[24]}$. Indeed, antiangiogenic strategies have long been studied and applied in different cancer settings ${ }^{[25]}$ and some drugs targeting the vascular endothelial growth factor (VEGF) such as the monoclonal antibody Bevacizumab (Avastin) are currently used in clinical therapy of gliomas with some success ${ }^{[26]}$. In this regard, strategies targeting the autocrine or paracrine loop of VEGF expression and secretion might be a plausible therapeutic approach. Interestingly, Rac1 is an important regulator of VEGF-mediated angiogenesis in different tumor types [27], including glioblastoma ${ }^{[28]}$.

Growing evidence indicates that a cellular subpopulation with stem cell like features, commonly referred to as glioma stem-like cells (GSLCs), is critical for tumor generation and maintenance. These GSLCs reside in stem-cell niches where they integrate extracellular signals (VEGF, IL-6, IL-8, IL-1beta), cell adhesion molecules and ECM components resulting in sustained growth and angiogenesis promotion.

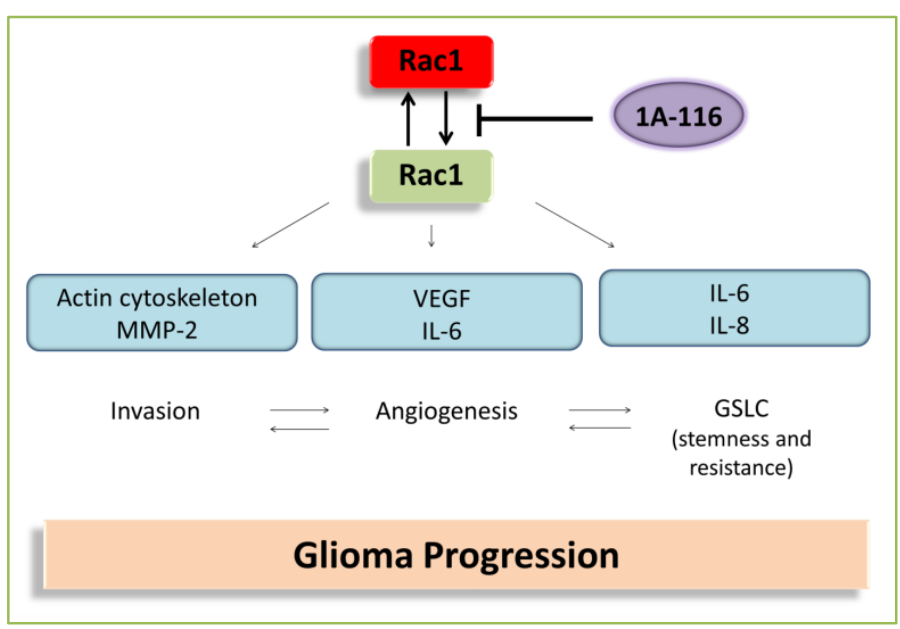

Figure 1. Rac1 signaling participates in the interplay of glioma cells with its brain microenvironment. Rac1 signaling pathway is involved in glioma invasion, angiogenesis and GSLCs maintenance via modulation of different molecular mechanisms. Rac1 inhibition by 1A-116 might be a plausible therapeutic approach to interfere glioma progression.

This cellular subpopulation is also involved in chemotherapy and radiotherapy resistance and contributes to glioma patients relapse ${ }^{[29]}$. In this regard, Rac1 has been described as a key molecule in maintenance of glioma cell stemness, conferring radiation and chemotherapy resistance in GSLCs [30].

Taken together, all these features show the intricate landscape in glioma progression and therapeutics. As schematized in Figure 1, glioma cell invasion, angiogenesis and the presence of GSLCs are interconnected and mutually dependent, supplying more levels of disease complexity. This dynamic interaction provides a new rationale for the development of novel anticancer strategies where heterogeneous features of glioma cells and their microenvironment should be targeted together to improve patient outcome. Rac1 stands out as an interesting target in this scenario, showing an important role in glioma cell behavior and its interaction with the ECM and its cellular components. There is an urgent need to validate Rac1 targeting in relevant preclinical models to further contribute with novel therapeutic options and try to restrain glioma progression.

\section{Conflicting Interest}

The authors declare that they have no Conflicting interests.

\section{Acknowledgements}

GA Cardama is a Research Fellow in ANPCyT and PL Menna is a member of the National Council for Scientific 
and Technical Research (CONICET). Our work has been supported by UNQ and Chemo-Romikin (Argentina).

\section{References}

1. Maher EA, Furnari FB, Bachoo RM, Rowitch DH, Louis DN, Cavenee WK, et al. Malignant glioma: genetics and biology of a grave matter. Genes Dev 2001;15:1311-1333.

2. Giese A. Glioma invasion--pattern of dissemination by mechanisms of invasion and surgical intervention, pattern of gene expression and its regulatory control by tumorsuppressor p53 and proto-oncogene ETS-1. Acta Neurochir Suppl 2003;88:153-162.

3. Etienne-Manneville S, Hall A. Rho GTPases in cell biology. Nature 2002;420:629-635.

4. Hall A. Rho GTPases and the actin cytoskeleton. Science 1998;279:509-514.

5. Salhia B, Tran NL, Symons M, Winkles JA, Rutka JT, Berens ME. Molecular pathways triggering glioma cell invasion. Expert Rev Mol Diagn 2006;6:613-626.

6. Hu B, Symons M, Salhia B, Fortin S, Tran N, Rutka J, et al. Rho GTPases and Their Activators, Guanine Nucleotide Exchange Factors (GEFs): Their Roles in Glioma Cell Invasion. In: Fatatis A, editor. Signaling Pathways and Molecular Mediators in Metastasis: Springer Netherlands; 2012. p. 143-169.

7. Salhia B, Tran NL, Chan A, Wolf A, Nakada M, Rutka F, et al. The guanine nucleotide exchange factors trio, Ect2, and Vav3 mediate the invasive behavior of glioblastoma. Am J Pathol 2008;173:1828-1838.

8. Jarzynka MJ, Hu B, Hui KM, Bar-Joseph I, Gu W, Hirose T, et al. ELMO1 and Dock180, a bipartite Rac1 guanine nucleotide exchange factor, promote human glioma cell invasion. Cancer Res 2007;67:7203-7211.

9. Vigil D, Cherfils J, Rossman KL, Der CJ. Ras superfamily GEFs and GAPs: validated and tractable targets for cancer therapy? Nat Rev Cancer 2010;10:842-857.

10. Cardama GA, Comin MJ, Hornos L, Gonzalez N, Defelipe L, Turjanski AG, et al. Preclinical development of novel Rac1-GEF signaling inhibitors using a rational design approach in highly aggressive breast cancer cell lines. Anticancer Agents Med Chem 2014;14:840-851.

11. Cardama GA, Gonzalez N, Ciarlantini M, Gandolfi Donadio L, Comin MJ, Alonso DF, et al. Proapoptotic and antiinvasive activity of Rac1 small molecule inhibitors on malignant glioma cells. Onco Targets Ther 2014;7:2021-2033.

12. Karnoub AE, Worthylake DK, Rossman KL, Pruitt WM, Campbell SL, Sondek J, et al. Molecular basis for Rac1 recognition by guanine nucleotide exchange factors. Nat Struct Biol 2001;8:1037-1041.

13. Gao Y, Xing J, Streuli M, Leto TL, Zheng Y. Trp(56) of rac1 specifies interaction with a subset of guanine nucleotide exchange factors. J Biol Chem 2001;276:47530-47541.

14. Wu X, Ramachandran S, Lin MC, Cerione RA, Erickson JW. A minimal Rac activation domain in the unconventional guanine nucleotide exchange factor Dock180. Biochemistry

\section{1;50:1070-1080.}

15. Feng H, Li Y, Yin Y, Zhang W, Hou Y, Zhang L, et al. Protein kinase A-dependent phosphorylation of Dock180 at serine residue 1250 is important for glioma growth and invasion stimulated by platelet derived-growth factor receptor alpha. Neuro Oncol 2015; $17: 832-842$.

16. Feng H, Hu B, Vuori K, Sarkaria JN, Furnari FB, Cavenee WK, et al. EGFRvIII stimulates glioma growth and invasion through PKA-dependent serine phosphorylation of Dock180. Oncogene 2014;33:2504-2512.

17. Aoki H, Yokoyama T, Fujiwara K, Tari AM, Sawaya R, Suki D, et al. Phosphorylated Pak1 level in the cytoplasm correlates with shorter survival time in patients with glioblastoma. Clin Cancer Res 2007;13:6603-6609.

18. Chan AY, Coniglio SJ, Chuang YY, Michaelson D, Knaus UG, Philips MR, et al. Roles of the Rac1 and Rac3 GTPases in human tumor cell invasion. Oncogene 2005;24:7821782-9.

19. Senger DL, Tudan C, Guiot MC, Mazzoni IE, Molenkamp G, LeBlanc R, et al. Suppression of Rac activity induces apoptosis of human glioma cells but not normal human astrocytes. Cancer Res 2002;62:2131-2140.

20. Fortin Ensign SP, Mathews IT, Symons MH, Berens ME, Tran NL. Implications of Rho GTPase Signaling in Glioma Cell Invasion and Tumor Progression. Front Oncol 2013;3:241.

21. Lorger M. Tumor microenvironment in the brain. Cancers (Basel) 2012;4:218-243.

22. Sun G, Cao Y, Shi L, Sun L, Wang Y, Chen C, et al. Overexpressed miRNA-137 inhibits human glioma cells growth by targeting Rac1. Cancer Biother Radiopharm 2013;28:327-334.

23. Wang M, Wang T, Liu S, Yoshida D, Teramoto A. The expression of matrix metalloproteinase-2 and -9 in human gliomas of different pathological grades. Brain Tumor Pathol 2003;20:65-72.

24. Das S, Marsden PA. Angiogenesis in glioblastoma. N Engl J Med 2013;369:1561-1563.

25. Garona J, Alonso DF. Reciprocal interactions between tumor and endothelial cells: effects of selective vasopressin V2 receptor peptide agonists. Cancer Cell \& Microenvironment 2014;1:1-5.

26. Seystahl K, Weller M. Is there a world beyond bevacizumab in targeting angiogenesis in glioblastoma? Expert Opin Investig Drugs 2012;21:605-617.

27. Ma J, Xue Y, Liu W, Yue C, Bi F, Xu J, et al. Role of activated Rac1/Cdc42 in mediating endothelial cell proliferation and tumor angiogenesis in breast cancer. PLoS One 2013;8:e66275.

28. Vader P, van der Meel R, Symons MH, Fens MH, Pieters E, Wilschut $\mathrm{KJ}$, et al. Examining the role of Rac1 in tumor angiogenesis and growth: a clinically relevant RNAi-mediated approach. Angiogenesis 2011;14:457-466.

29. Persano L, Rampazzo E, Basso G, Viola G. Glioblastoma cancer stem cells: role of the microenvironment and therapeutic targeting. Biochem Pharmacol 2013;85:612-622.

30. Yoon CH, Hyun KH, Kim RK, Lee H, Lim EJ, Chung HY, et al. The small GTPase Rac1 is involved in the maintenance of stemness and malignancies in glioma stem-like cells. FEBS Lett 2011;585:2331-2338. 